\title{
Las tres generaciones de la UAL (lipoplastia asistida por ultrasonido)
}

\author{
The three generations of the UAL \\ (ultrasound assisted lipoplasty)
}

\author{
Roberto Rodrigo Cáceres
}

\section{ÍNDICE}

1. Resumen/Summary .3

2. Introducción............................................................... 4

La historia de la liposucción........................................ 6

El ultrasonido en la lipoplastia.................................... 7

Evolución de las generaciones de la UAL ................ 8

Física básica de la UAL ............................................... 9

Fundamentos del Efecto de la UAL ....................... 14

Efectos de los diseños de cánulas y sondas ...........16

Efecto de la UAL sobre los tejidos.......................... 19

3. Materialesy métodos .................................................... 18

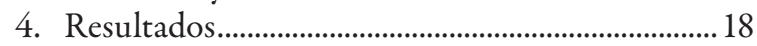

5. Discusión ....................................................................19

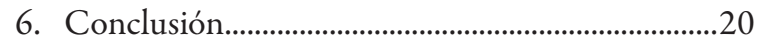

7. Anexos ...........................................................................

8. Bibliografía ....................................................................23

\section{RESUMEN}

La Lipoplastia asistida por ultrasonido (UAL) es una técnica empleada desde ya muchos años. Con el desarrollo de nuevas tecnologías, se replantea constantemente la terapéutica más eficaz con los nuevos recursos disponibles, por lo que se elaboran aparatos capaces de optimizar resultados llevando a un tratamiento más innovador y eficaz.

Surgen nuevos conocimientos sobre las propiedades físico-mecánicas de los aparatos con ultrasonido, lo cual abre una ventana de oportunidades para su uso médico, particularmente para el uso en la liposucción en el tratamiento de los pacientes con lipodistrofia corporal,

1. Rotación: Cirugía Estética. Especialista en Cirugía Plástica y Reconstructiva, Especialista en Cirugía General. Universidad del Salvador.

$\triangle$ Correspondencia: Dr. Roberto Rodrigo Cáceres. cacerescaceres. rodrigo@usal.edu.ar

Los autores no declaran conflictos de intereses

Recibido: 09/09/2018 / Aceptado: 21/09/2018 quienes podrían ser beneficiados por las ventajas de estas tecnologías en comparación con las que actualmente están en uso.

Las características de los aparatos de ultrasonido en el campo de la cirugía plástica y su evolución con las décadas ha permitido obtener mayor eficacia en su acción y ademas ha disminuido las complicaciones. Se realiza una recopilación de datos sobre la Liposucción Asistida por Ultrasonido en el tratamiento de la lipodistrofia. Se exponen las cualidades del aparato ultrasónico en la lipodistrofia, su historia evolutiva, sus logros y mejores resultados, anteriormente no alcanzados con las técnicas hasta ahora empleadas.

Palabras claves: lipoplastia, ultrasonido, lipodistrofia.

\section{SUMMARY}

Ultrasound-assisted Lipoplasty (UAL) is a technique that has been used for many years. With the development of new technologies, the most effective therapy is constantly being rethought with the new resources available, so that devices are developed capable of optimizing results leading to a more innovative and effective treatment.

New knowledge about the physico-mechanical properties of ultra sound devices emerges, which opens a window of opportunities for medical use, particularly for the use in liposuction the treatment of patients with body lipodystrophy, who could benefit from the advantages of these technologies compared to those currently in use.

Due to the characteristics of ultrasound devices in the field of plastic surgery and its evolution over the decades, it has allowed greater effectiveness in its action, in addition to reducing complications. A collection of data on Ultrasound-Assisted Liposuction is performed in the treatment of lipodystrophy.

The qualities of the ultrasonic apparatus in lipodystrophy, its evolutionary history, its achievements and better results, previously unreached with the techniques used until now, are exposed.

Keywords: lipoplasty, ultrasound, lipodystrophy. 


\section{INTRODUCCION}

\subsection{LA HISTORIA DE LA LIPOSUCCIÓN}

Las raíces de la liposucción se remontan a la década de $1920^{1}$. El primer intento para eliminar la grasa a través de pequeñas incisiones se describió en 1929. Un cirujano francés, Charles Dujarrier, intentó mejorar las pantorrillas y las rodillas de un bailarín de ballet utilizando una cureta para eliminar la grasa. Se produjo un resultado desafortunado, trágicamente daño a los vasos sanguíneos más grandes lo que provocó la amputación de una pierna ${ }^{2}$. Los siguientes intentos documentados para eliminar grasa mediante pequeñas incisiones se realizaron en 1968 por el médico estadounidense Wilkinson y en 1972 por el cirujano alemán Schrudde, quien describió por primera vez el uso de una cureta de aspiración ${ }^{2}$. Sin embargo, en ambos casos, el método de tratamiento pronto fue abandonado debido a los extensos efectos secundarios. Otros cirujanos durante la década de 1960 intentaron mejorar la forma del cuerpo mediante la eliminación de la grasa subcutánea. Se intentó tanto el legrado como la remoción en bloque, pero sin buenos resultados estéticos ${ }^{3}$.

Los pioneros generalmente aceptados de la forma moderna de la liposucción son Giorgio Fischer y su padre Arpad Fischer. A mediados de la década de 1970, desarrollaron un instrumento el cellusuctiotome que consiste en una cureta hueca con una cuchilla, unida a una bomba de succión ${ }^{4}$. La cureta separó la grasa que se aspira al cortarla con una cuchilla accionada por motor. La grasa fue transportada a través de un sistema de tubos. Este método tuvo una alta tasa de complicaciones en forma de hemorragia, hematoma posoperatorio, seroma y formación de hendiduras ${ }^{4}$.

Yves-Gerard Illouz, de París, hizo importantes avances en el desarrollo de la liposucción, convirtiéndola en un procedimiento seguro y de rutina. Illouz reemplazó la cureta de bordes afilados con una cánula roma, menos traumática. De hecho, usó un sistema de succión que hasta entonces solo se había usado en el campo ginecológico para las terminaciones del embarazo: el aspirador Karman con cánulas uterinas. Este equipo fue mucho más fácil de obtener para los colegas interesados que el prototipo diseñado por Fischers, lo que resultó en la popularización de este método. Illouz desarrolló aún más el concepto de tratar el tejido en varias capas, utilizando lo que se conoce como la "técnica húmeda". Esto implicó la inyección de solución salina mezclada con hialuronidasa en el tejido adiposo antes del tratamiento. A través de estas mejoras, fue posible reducir la tasa de complicaciones considerablemente ${ }^{5}$.

Pierre Fournier ha hecho mucho para desarrollar y promover la liposucción moderna ${ }^{6}$. Fournier mostró un temprano interés en la técnica de lipoescultura de Fischers y ayudó a promover técnicas modernas de liposucción para médicos de todo el mundo. Pierre Fournier dio un gran impulso al desarrollo de la técni-

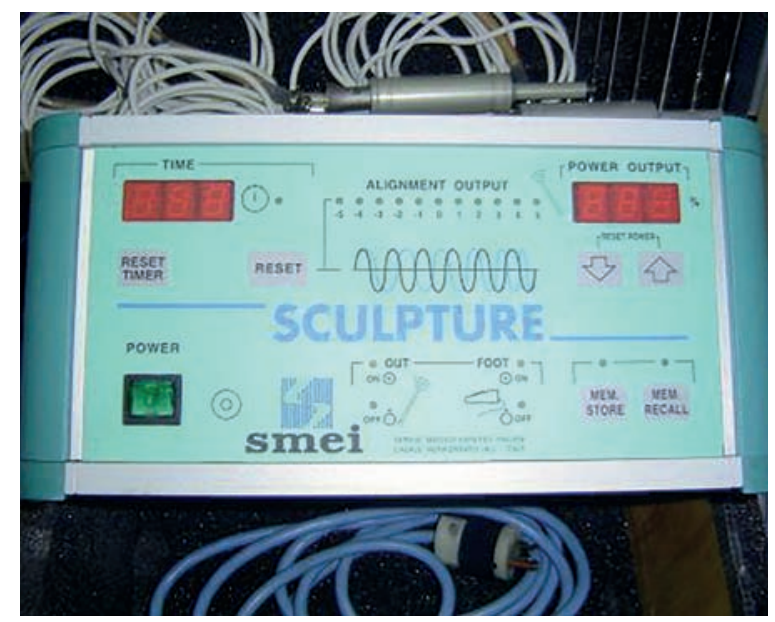

Figura 1. Primera generación de ultrasonidos. Sculpture System. https://www.dotmed.com/listings/print/print.html?id=689558

ca de liposucción para evitar resultados operativos no uniformes. Recomendó el uso de la técnica 'cruzada', que consistía en superposición de tratamientos del área a aspirar en varios niveles, desde varias incisiones y desde varios ángulos. Para lograr un efecto crioanestésico, inyectó solución salina enfriada después de la adopción de la "técnica húmeda"

A mediados de la década de 1980, Fournier publicó su trabajo sobre la técnica de transferencia de grasa como un medio de aumento de tejido. Esto implicó la extracción de la grasa, primero con microcánulas y más tarde con cánulas de mayor diámetro, unidas a una jeringa. Fournier también abogó por la aspiración manual de grasa, utilizando jeringas, para modelar el cuerpo, lipoescultura asistida por jeringas $(\mathrm{LAL})^{8}$.

Otro hito en el desarrollo de la cirugía de liposucción moderna fue la introducción en 1985 de la anestesia tumescente por Jeffrey Klein, quien publicó por primera vez el procedimiento en 1987. La técnica tumescente permitió el rendimiento de más procedimientos extensivos de liposucción bajo anestesia local de forma ambulatoria. Al mismo tiempo, debido a la interacción farmacológica y física específica con el tejido a tratar, la liposucción fue considerablemente más segura y permitió un mayor desarrollo 9 . Debido a la baja tasa de complicaciones y los excelentes resultados cosméticos, la liposucción por anestesia local tumescente es actualmente el método de elección ${ }^{10}$.

La instrumentación ultrasónica para la aplicación quirúrgica se introdujo por primera vez para el descascarillado de la placa dental a fines de la década de 1950 y principios de la de $1960^{11}$. En 1969, Kelman adaptó la sonda de metal vibrante al procedimiento de facoemulsificación. La capacidad de aspiración e irrigación se agregó al dispositivo vibratorio básico para facilitar la extracción segura y efectiva de la catarata, utilizando esta tecnología y la técnica asociada. El efecto clínico del dispositivo de facoemulsificación en la catarata se ha atribuido a un efecto de microcorte ${ }^{12}$.

En 1974, el dispositivo de facoemulsificación se modificó 


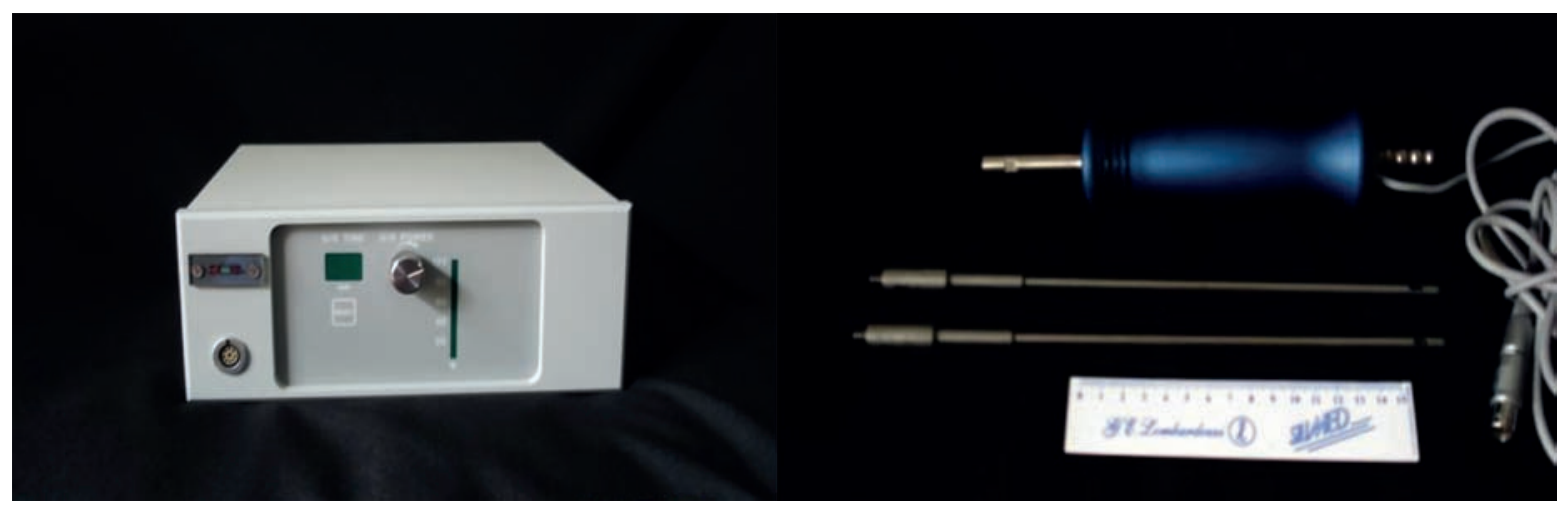

Figura 2. Primera generación de ultrasonidos. Emulsifier. Hospital Británico de Bs.As.Curso: Contorno Corporal de Alta Definición con Ultrasonido. Mayo 2006. Dr Eduardo Marchioni.

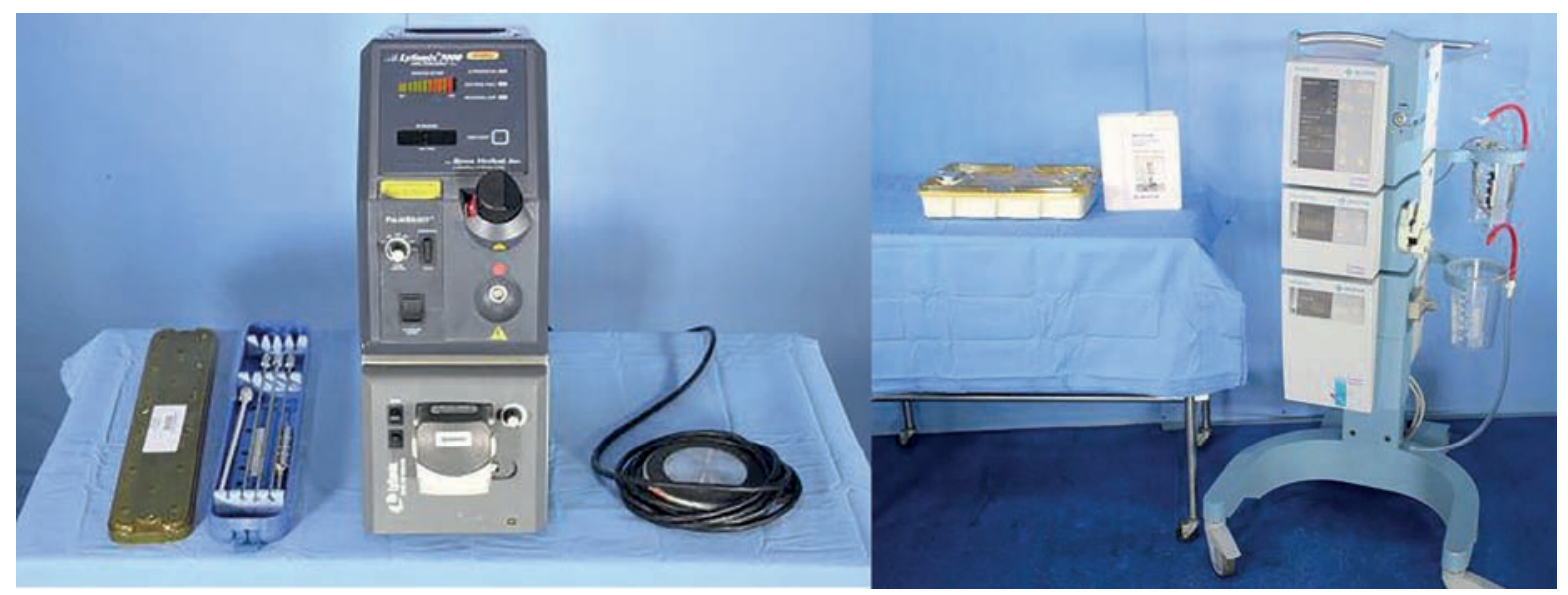

Figura 3. Segunda generación de Ultrasonidos. Lysonix 3000, y Contour Genesis. https://www.dotmed.com/listing/ultrasonic.

aún más y se aplicó a la neurocirugía para la extirpación del tumor. El objetivo del dispositivo era eliminar selectivamente los tejidos patológicos del cerebro y de la columna vertebral con un mínimo trauma residual en los tejidos restantes. El dispositivo CUSA (aspirador quirúrgico ultrasónico cavitrón). La naturaleza selectiva del tejido de los dispositivos, es decir, la capacidad de preservar los nervios y los vasos, se ha atribuido a la capacidad del dispositivo para diferenciar entre los tejidos con diferentes contenidos de agua y un proceso denominado cavitación ${ }^{13}$. A fines de los años ochenta y principios de los noventa, el concepto de instrumento ultrasónico se adaptó a un dispositivo de corte y coagulación con aplicación a la cirugía laparoscópica en el abdomen ${ }^{14}$.

La aplicación de instrumentación ultrasónica para la cirugía de contorno corporal comenzó a finales de los años ochenta y principios de los noventa. Scuderi ${ }^{15} y$ Zocchi $^{16}$ fueron pioneros en la aplicación de la vibración ultrasónica a la emulsificación y eliminación de grasas. La esperanza y el objetivo de este esfuerzo fue crear tecnología y técnicas asociadas que producían consistentemente un medio más seguro y más efectivo de contorno corporal estético en comparación con los métodos conocidos de la época, a saber, la lipoplastia asistida por succión (SAL). Se esperaba que los beneficios de la selectividad tisular demostrados y utilizados en las aplicaciones quirúrgicas mencionadas an- teriormente, especialmente la neurocirugía, produjeran un método de lipoplastia que fuera más "específico de grasa" que la cánula de succión existente y conocida. Esta tecnología y técnica se denominó lipoplastia asistida por ultrasonido (UAL) ${ }^{17}$.

Con los primeros usos de la tecnica UAL se demostró algunas desventajas significativas: requirió cánulas relativamente grandes, y se registraron tasas relativamente altas de seroma, quemaduras en la piel, hiperestesia posoperatoria persistente e hiperestesia como resultado del daño a las lipoproteínas de las envolturas neurales ${ }^{18}$.

Para disminuir las desventajas que presentaba la técnica de liposucción asistida por ultrasonido, fueron estudiados más profundamente los mecanismos fisico-mecanico de los aparatos, por lo que evolucionaron con los años. Así, cada generación ha mejorado, demostrando una mayor efectividad, con menores complicaciones, al tiempo de una menor utilización de energía para lograr el efecto deseado. Los dispositivos de lipoplastia ultrasónica de primera generación llegaron a finales de la década de 1980, dispositivos de segunda generación a mediados de la década de 1990 y tercera generación a principios de la década de $2000^{14}$.

\subsection{EL ULTRASONIDO EN LA LIPOPLASTIA}

El uso de vibraciones de alta frecuencia en instrumentos quirúrgicos, comúnmente conocidos como instru- 

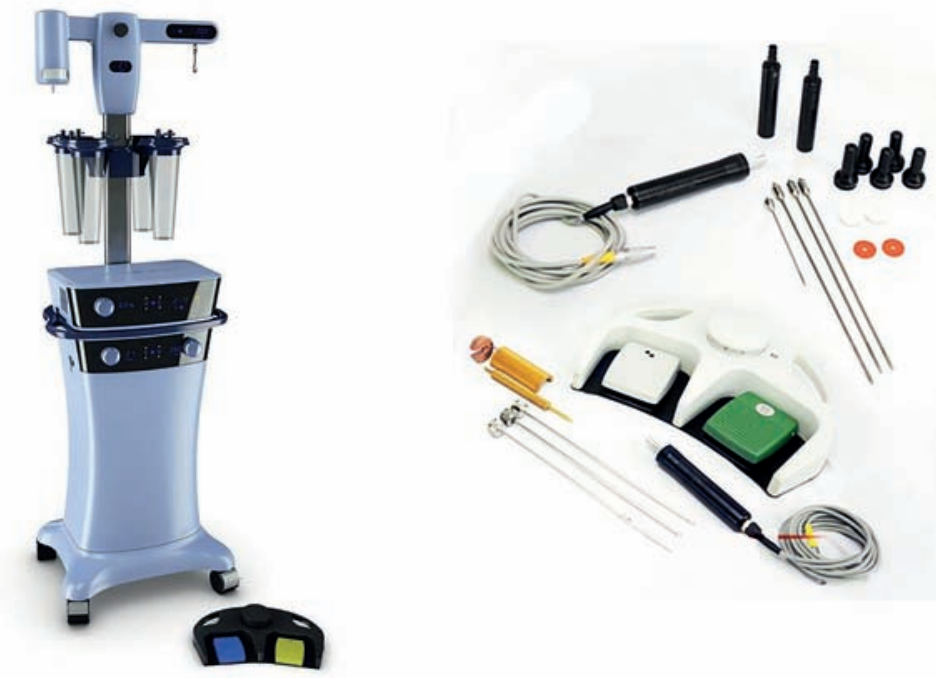

Figura 4. Tercera generación de Ultrasonidos. VASER. http://www.vaser.com/vaserphysician/vaser-products/ventx-console

mentación quirúrgica ultrasónica, generalmente implica una frecuencia de vibración en el rango de 20,000 ciclos por segundo $(20 \mathrm{kHz})$ a 60,000 ciclos por segundo $(60 \mathrm{kHz})$. La punta de metal o punta del instrumento quirúrgico se mueve hacia adelante y hacia atrás a las frecuencias antes mencionadas para crear un efecto quirúrgico deseado. La elección de la frecuencia y el diseño de la punta de la sonda de metal determinan la aplicación del instrumento y la forma en que el dispositivo interactúa con el tejido objetivo ${ }^{14}$.

La liposucción asistida por ultrasonido (UAL) es una forma de liposucción que emplea energía ultrasónica para permitir una lipólisis tisular más selectiva, Zocchi describió inicialmente la técnica, donde utilizó un proceso de dos etapas de lipólisis selectiva del tejido utilizando una sonda ultrasónica seguida de lipectomía asistida por succión (SAL) tradicional para evacuar la grasa ${ }^{19}$. La lipólisis adiposa selectiva observada en UAL se puede atribuir a la transformación de la energía eléctrica en energía mecánica a través de una sonda ultrasónica que vibra a frecuencias superiores a 16 $\mathrm{kHz}$. Esta onda de sonido oscilante produce un vector de presión negativa que supera selectivamente las fuerzas moleculares dentro del tejido adyacente y conduce a la cavitación y fragmentación celular ${ }^{20}$.

La disrupción celular a gran escala se aspira posteriormente en una segunda etapa de liposucción. Rohrich et al, confirmaron que UAL genera niveles significativamente altos de varias enzimas adipocíticasintracelulares, más que la SAL, lo que confirma la noción de que los adipocitos se lisan mecánicamente en grandes cantidades ${ }^{21}$.

Uno de los beneficios reportados de la UAL sobre otras formas de lipoplastia asistida por succión asistida por energía y térmica es la formación de cavitación del aire burbujeado en el fluido tumescente. Este proceso da como resultado un efecto de "barra de cuerdas" de transmisión de células de grasa intactas y células madre derivadas de adipocitos para que luego puedan usarse para el injerto de grasa exitoso ${ }^{22}$.

Los partidarios de UAL afirman que la cavitación de los adipocitos antes de la SAL reduce la pérdida de sangre y el tiempo quirúrgico, al tiempo que proporciona menos equimosis y molestias, así como un mejor contorno en áreas con abundante tejido fibroso, como la espalda y el tórax ${ }^{23}$.

Las evidencias para justificar las afirmaciones de disminución del tiempo operatorio y la equimosis son mixtas, varios estudios han confirmado que la UAL es efectiva en casos de liposucción secundaria y en la liposucción en áreas fibróticas como el flanco masculino o el área del tórax. Beckenstein y Grote demostró que la UAL fue efectiva en el 70\% de los casos de lipoescultura secundaria ${ }^{24}$. Además, Fodor y Watson compararon directamente a UAL con SAL y respaldaron la superioridad de UAL en esculpir áreas fibróticas en comparación con SAL ${ }^{25}$.

\subsection{EVOLUCIÓN DE LAS GENERACIONES DE LA UAL} El dispositivo UAL de primera generación fue producido por la Compañía SMEI de Italia, Sculpturesystem (Figuras 1, 2). Utilizó sondas lisas y sólidas a una frecuencia de $20 \mathrm{kHz}$. Las sondas sólidas tenían un diseño escalonado con diámetros en la punta de tan solo $3,0 \mathrm{~mm}$ (sonda pequeña) y diámetros en la base de hasta 6,0 $\mathrm{mm}$ (sonda grande). La técnica básica implicaba una buena práctica quirúrgica y dos reglas fundamentales: (1) el uso esencial de un entorno húmedo producido por la infiltración de suficiente solución anestésica húmeda , y (2) el movimiento constante de la son- 


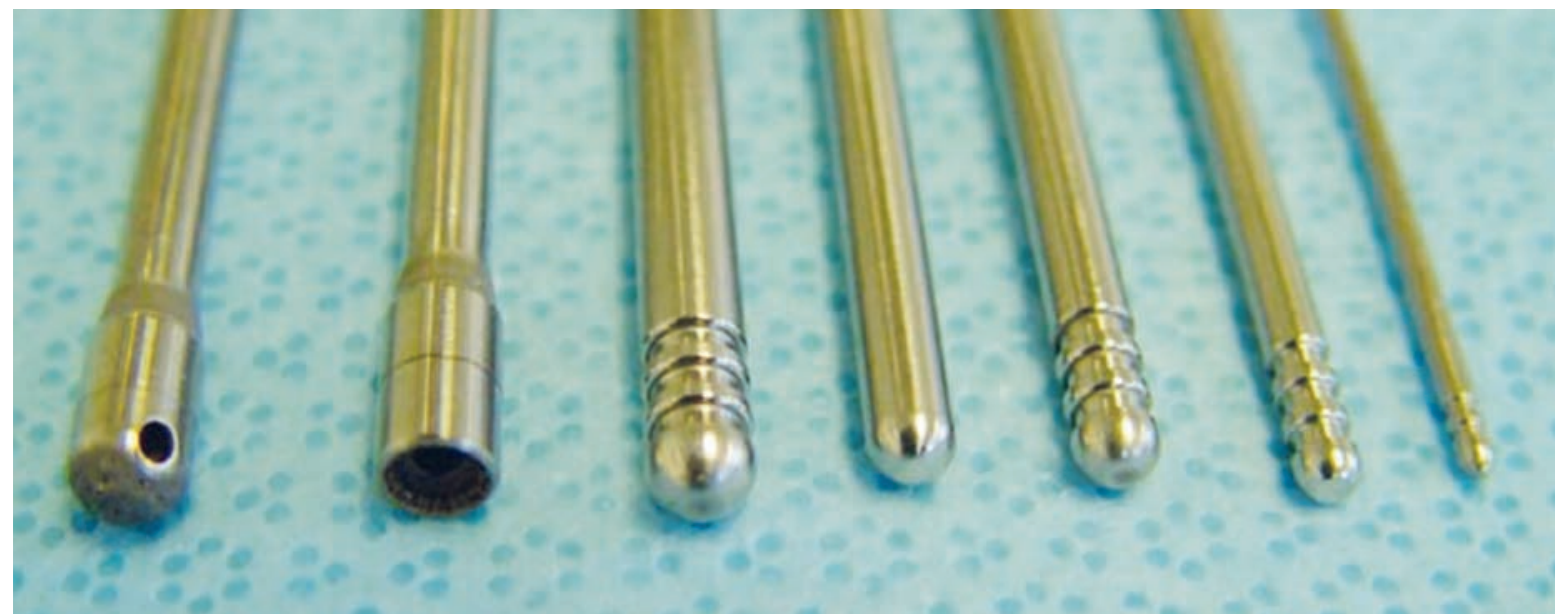

Figura 5. Después de la emulsion blanda, las estructuras de colágeno, los vasos y el tejido nervioso se salvan. (Cimino, W.William; Shiffman, Melvin A. Ultrasound-Assisted Lipoplasty: Basic Physics, Tissue Interactions, and Related Results/Complications. Body Contourning. 2010: 394.)

da para evitar daños térmicos ${ }^{26}$. Los tiempos quirúrgicos iniciales estuvieron en el rango de 10 a 12 min para una remoción de 250-300 $\mathrm{ml}$ o aproximadamente 4 minutos de ultrasonido por cada $100 \mathrm{ml}$ de aspirado ${ }^{26}$ Alrededor de 1995, un creciente interés en UAL en los Estados Unidos llevó al liderazgo comunitario de cirugía plástica a crear una Fuerza de Tarea UAL que incluía representación de la Sociedad Estadounidense de Cirugía Plástica Estética, Fundación de Investigación Educativa de la Sociedad Estética, Sociedad Americana de Cirugía Plástica, Sociedad de Lipoplastia de América del Norte y PlasticSurgeryEducationFoundation. La misión de la TaskForce era evaluar la nueva instrumentación ultrasónica para la lipoplastia y ayudar en su enseñanza e introducción en los Estados Unidos. Los cursos de enseñanza se ofrecieron bajo la supervisión de la TaskForce con capacitación didáctica y práctica ${ }^{25}$

Durante el período de TaskForce UAL, los dispositivos UAL de segunda generación estuvieron disponibles a mediados de los 90. Estos dispositivos incluyen Lysonix 2000 (Lysonix Inc., Carpinteria, CA) y Mentor Contour Genesis (Mentor Corporation, Santa Barbara, CA), (Figura 3). El sistema Lysonix funcionaba a una frecuencia de $22.500 \mathrm{~Hz}(22,5 \mathrm{kHz})$ y utilizaba cánulas ultrasónicas huecas que aspiraban grasa emulsionada simultáneamente con el proceso de emulsión. Las ofertas de cánula eran diseños de "golf-tee" y "bullet" con diámetros de 4.0 y $5.1 \mathrm{~mm}$. El diseño de la punta "golf-tee" con un diámetro de $5.1 \mathrm{~mm}$ fue, en teoría, el diseño más utilizado debido a su eficiencia más alta. El Mentor Contour Genesis era un sistema integrado con succión, infusión y un generador ultrasónico, todo en un solo sistema móvil. La frecuencia ultrasónica fue de $27,000 \mathrm{~Hz}(27,0 \mathrm{kHz})$ y también se utilizó una cánula ultrasónica hueca, con diámetros ofrecidos de 3,0 a 5,1 mm. La forma de la punta de las cánulas Mentor era plana con orificios laterales para la aspiración de todos los diámetros de punta ${ }^{14}$.
La técnica UAL continuó evolucionando con los dispositivos Lysonix y Mentor. Originalmente, los tiempos de aplicación eran largos, y se informaron complicaciones significativas, y se cuestionó la seguridad ${ }^{27}$. A medida que se redujeron los tiempos de aplicación, la tasa de complicaciones disminuyó. Los tiempos de aplicación se redujeron a 1 minuto de ultrasonido por $100 \mathrm{ml}$ de aspirado ${ }^{28}$. El concepto de "pérdida de resistencia" se hizo ampliamente conocido como un punto final quirúrgico realístico.

El movimiento rápido de la sonda se introdujo como otro medio para controlar de forma segura la energía presentada por las máquinas de segunda generación. En general, los resultados variaron en un extremo desde el uso seguro y efectivo de UAL hasta las altas tasas de complicaciones y la seguridad cuestionable en el otro extremo ${ }^{29}$.

A principios de 2001, apareció una tercera generación de instrumentos ultrasónicos para cirugía de contorno corporal. Esta tecnología fue nombrada VASER, para la amplificación de la vibración de la energía del sonido en Resonancia, (Figura 4). La tecnología VASER y la técnica asociada (SoundSurgical Technologies, Louisville, CO), conocida como VAL para Lipoplastia asistida por VASER, se diseñó para minimizar o eliminar las complicaciones conocidas de generaciones anteriores de tecnología UAL y para darse cuenta simultáneamente de los beneficios de la instrumentación ultrasónica establecida en otros escenarios quirúrgicos ${ }^{14}$.

El concepto rector fue desarrollar instrumentación que emulsionara el tejido adiposo de forma rápida y segura con la cantidad mínima absoluta de energía, logrando así el resultado deseado con poco o ningún trauma residual en los tejidos restantes. La instrumentación VASER introdujo los conceptos de suministro pulsado de energía ultrasónica, sondas sólidas de pequeño diámetro $(2.2-3.7 \mathrm{~mm})$ y diseños de sonda acanalada para aumentar la eficiencia. Se introdujeron cánulas de emul- 
sión suaves para la fase de aspiración para preservar la delicada estructura de la matriz tisular después de que se completara el proceso de emulsión ${ }^{14}$

En 1999 y 2001, Cimino publicó los primeros estudios científicos que definían la cantidad de energía suministrada a los tejidos por diversos dispositivos quirúrgicos ultrasónicos y definía claramente las variables bajo el control del cirujano que determinan la seguridad y los resultados ${ }^{30}$. Esta información científica básica condujo a relaciones claramente entendidas entre "causas" y "efectos" cuando se usa instrumentación quirúrgica ultrasónica para la cirugía de esculpido corporal. Como consecuencia directa, el aspecto de succión de la instrumentación ultrasónica fue eliminado (cánulas ultrasónicas huecas) y reemplazado por diseños de sonda sólida, los diámetros de la sonda se redujeron significativamente, la eficiencia se mejoró utilizando los diseños de sonda ranurada y se introdujo la energía pulsa$\mathrm{da}$, todo lo cual redujo significativamente la energía entregada al paciente. Un estudio clínico piloto en $77 \mathrm{pa}-$ cientes completado por Jewell et al. utilizando el VASER y la técnica VAL mostró cero complicaciones y resultados efectivos ${ }^{31}$.

\subsection{FÍSICA BÁSICA DE LOS UAL}

El uso seguro y eficaz de la instrumentación ultrasónica para la lipoplastiarequiere una comprensión tanto de la tecnología como de los métodos quirúrgicos asociados que difieren significativamente en muchos aspectos de las herramientas y los métodos básicos de la lipoplastia asistida por succión. Se discutirán las interacciones físicas y tisulares básicas para la realización de la lipoplastia asistida por ultrasonido, los beneficios del uso apropiado de esta tecnología y las complicaciones asociadas con su uso incorrecto.

El sistema básico de cirugía ultrasónica tiene un generador electrónico que interactúa con una pieza de mano ultrasónica. La pieza de mano ultrasónica tiene un motor ultrasónico, la mayoría de las veces compuesto de cristales piezoelectrico, PZT (titanato zirconato de plomo ) que convierten la energía eléctrica en movimiento vibratorio. El movimiento vibratorio se pasa a una sonda que vibra en resonancia con la pieza de mano. Los circuitos electrónicos en el generador mantienen la vibración a la frecuencia de resonancia seleccionada y ajustan la amplitud de la vibración según los controles en el generador ${ }^{13}$.

Las frecuencias de vibración para los sistemas ultrasónicos para lipoplastia oscilan entre 22 y $36 \mathrm{kHz}$. Se puede afirmar que a menor oscilacion, se produce una mayor interacción con los tejidos de alta densidad como el tejido conectivo, muscular, osea. Y a mayor oscilacion se encuentra la especificidad con el tejido adiposo. Se altera las longitudes de las piezas resonantes al cambiar la longitud de onda de la vibración ${ }^{14}$.

Las sondas ultrasónicas vibran con las ondas estacionarias y así logran la capacidad de concentrar energía en la punta de la sonda. Las ondas estacionarias se establecen en la sonda y la pieza de mano de modo que la punta de la sonda experimente un movimiento longitudinal máximo, del orden de un desplazamiento pico a pico de unas pocas milésimas de pulgada, apenas visible a simple vista. La amplitud es una función de la frecuencia de vibración y la configuración de la energía. Es importante entender que la vibración no es "lateral", es decir, transversal al eje largo de la sonda. Cuando se produce una vibración transversal, como a veces ocurre con un diámetro más pequeño o sondas más largas, tiene un zing audible muy fuerte. Dicha vibración puede fracturar fácilmente la sonda ultrasónica porque la sonda no fue diseñada para soportar tensiones de flexión ${ }^{14}$.

La potencia eléctrica en el generador o la amplitud de la vibración no es un indicador útil de la potencia real que afecta a los tejidos. La energía depositada en los tejidos es una función del ajuste del generador, y también una función fuerte del "acoplamiento" entre la punta de la sonda y el tejido. Una punta vibratoria que se presiona fuertemente en el tejido juntará significativamente más energía al tejido que la misma punta que toca suavemente el mismo tejido. Además, el diseño y la forma de la punta vibratoria influirán en gran medida en la medida de la potencia que se acopla al tejido y el punto en la punta donde se concentrará la energía. Por lo tanto, lo que se necesita es una medida de la potencia acústica máxima que se podría acoplar a partir de una sonda con un diseño específico y una amplitud de vibración seleccionada. Esta información ha sido medida e informada ${ }^{30}$.

Se demostró que los dispositivos ultrasónicos de primera y segunda generación, cuando se ejecutan en el rango de amplitudes clínicamente efectivas, entregan entre 20 y $30 \mathrm{~W}$ de potencia al contacto con agua. Los dispositivos de tercera generación normalmente entregan 10-15 vatios de potencia al contacto con agua; generalmente el 50\% de la potencia de los dispositivos de generación anterior ${ }^{30}$.

\subsection{FUNDAMENTOS DEL EFECTO DE LA UAL}

La interacción entre la punta de la sonda ultrasónica y el tejido es una función compleja de tres fenómenos diferentes. Las tres interacciones básicas de tejido son: (1) cavitación, (2) térmica y (3) mecánica.

La teoría de la cavitación esta dada por la interacción entre el dispositivo ultrasónico y el tejido adiposo. La teoría sostiene que la energía ultrasónica en la punta de la sonda induce un campo acústico que hace que los gases disueltos en el tejido y los fluidos se acumulen en burbujas que luego son accionadas acústicamente para crecer en tamaño hasta que se vuelven inestables y en ese momento implosionan. La implosión es un proceso violento que libera energía en forma de ondas de choque y calor. La implosión en realidad libera solo una cantidad muy pequeña de energía por burbuja 


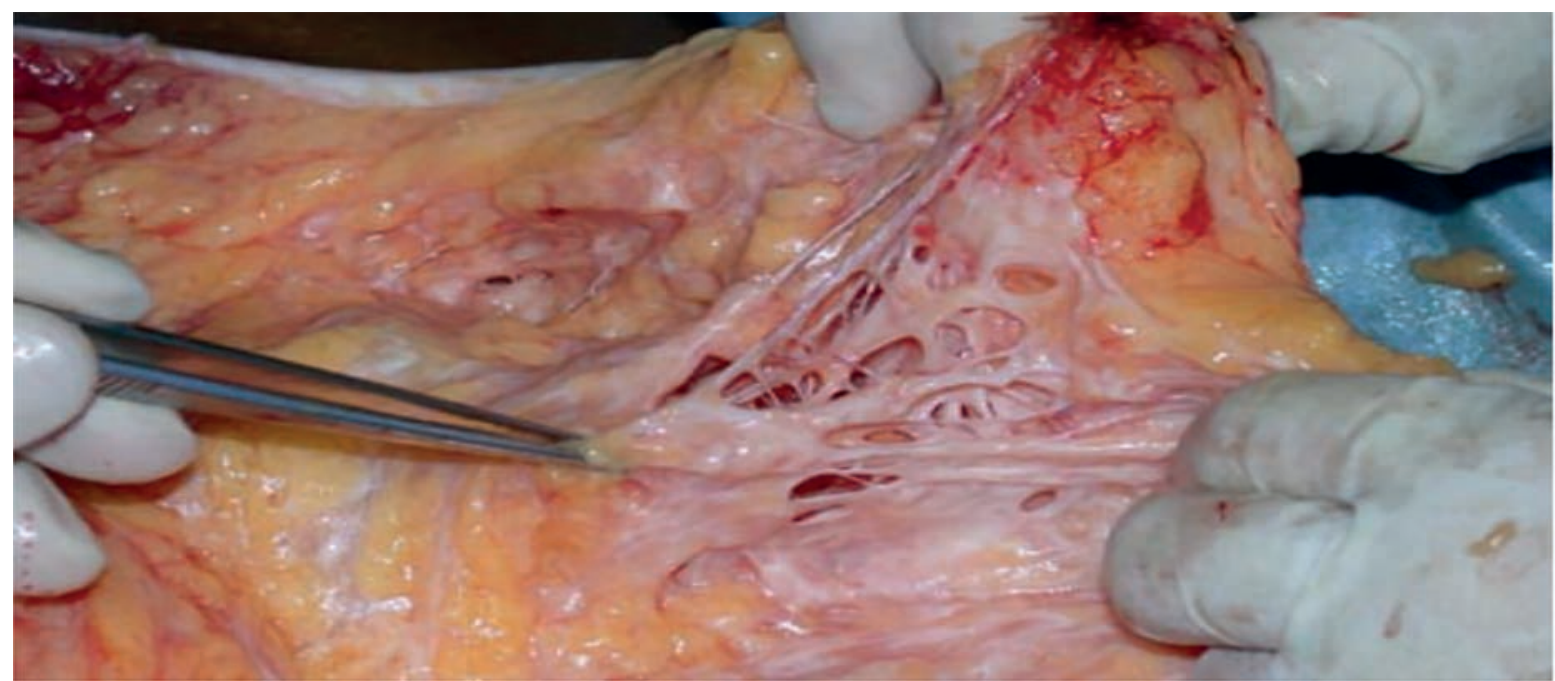

Figura 6. Diferentes modelos de cánulas y sondas, según las evoluciones de las generaciones (Cimino, W.William; Shiffman, Melvin A. Ultrasound-Assisted Lipoplasty: Basic Physics, Tissue Interactions, and Related Results/Complications. Body Contourning. 2010: 394.)

porque las burbujas son muy pequeñas. La energía neta es la suma de las muchas burbujas que se generan por la energía ultrasónica en la punta de la sonda. Las burbujas de cavitación existen como una "barba" alrededor de la punta de la sonda ultrasónica, aparentemente unida a la superficie de la sonda y extendiéndose a no más de un milímetro de la misma. Las burbujas existen en los cambios de perfil en la punta, no a lo largo de las superficies lisas paralelas al eje de vibración. Aunque la cavitación tiene un efecto notorio, no es la interacción tisular predominante ${ }^{32}$.

La teoría térmica sostiene que la energía ultrasónica esencialmente "derrite" el tejido adyacente. Ciertamente, es posible generar calor con un dispositivo de sonda ultrasónica. Sin embargo, esto es lo opuesto a los objetivos quirúrgicos y del tratamiento. La adición de grandes cantidades de solución anestésica húmeda y el movimiento adecuado de la sonda garantizarán que no se genere calor significativo. Alguna pequeña cantidad de calor siempre será generada por una sonda vibratoria de alta frecuencia. Sin embargo, la cantidad y la distribución de la energía térmica se pueden controlar y gestionar fácilmente de manera que el proceso de emulsificación pueda tener lugar sin efectos térmicos adversos. La cantidad de generación de calor ha sido medida y cuantificada ${ }^{30}$.

La teoría mecánica sostiene que cuando la punta de metal que se mueve rápidamente de la sonda ultrasónica se encuentra con el tejido, crea un impacto inducido por la vibración de alta energía y condiciones de flujo que fragmentan el tejido adiposo. Las presiones altas y bajas, la transmisión acústica rápida y los impactos con superficies metálicas de movimiento rápido, individualmente y en combinación, son suficientes para fragmentar el tejido ${ }^{33}$.

Es probable que todos los tres tipos de interacciones estén presentes en la mayoría de las situaciones, en dis- tintos grados. El diseño de la instrumentación y la técnica utilizada por el cirujano influirán en qué parte de cada interacción está presente. Con respecto al diseño, las sondas que se ejecutan en amplitudes excesivas, o que tienen formas globales lisas, son ineficientes y darán como resultado más deposición de energía térmica y menos fragmentación mecánica. Las sondas con superficies frontales planas o cóncavas generarán una energía de cavitación excesiva que finalmente se convertirá en energía térmica y también tendrá densidades de energía muy altas a lo largo de estas superficies. La eficiencia mecánica se optimiza con diseños de sonda con muchas superficies perpendiculares al eje de vibración y la eliminación de bordes filosos ${ }^{14}$.

Con respecto a la técnica quirúrgica, el uso de suficiente solución anestésica húmeda y el movimiento constante de la sonda eliminarán los problemas térmicos. La punta vibratoria no debe presionarse fuertemente en ningún tejido ya que esto elimina el fluido protector y acopla fuertemente la energía ultrasónica al tejido, lo que resulta en una fuerte deposición de energía térmica (golpe final). Deben evitarse las sondas de gran diámetro ya que poseen una energía vibratoria excesiva y requieren un "empuje" significativo para atravesar el tejido a menos que la amplitud se eleve demasiado, lo que da como resultado una energía vibratoria excesiva aplicada a los tejidos ${ }^{14}$.

\subsection{EFECTOS DE LOS DISEÑOS DE LAS CÁNULAS O SONDAS DE LOS UAL}

Hay una variedad de diseños de sondas y cánulas que se pueden usar (Figura · 5). Con el equipo de primera generación, apareció la primera sonda, presentaba una punta hemisférica lisa la cual era rígida, sin orifico, por lo tanto producía un gran efecto sobre los tejidos con los que entraba en contacto, con esta se observaba muchos efectos colaterales por la eficiencia baja de dicho 
diseño y la intensidad energética en el área activa. Con la segunda generación aparecieron las cánulas de superficie frontal plana con dos orificios laterales de aspiración; en este caso la mayoría de la superficie frontal está activa en esta sonda. Por lo tanto, cuando la sonda se presiona fuertemente en los tejidos, hay un fuerte acoplamiento de la energía ultrasónica desde la cara de la sonda. El otro diseño de cánulas es en "soporte de golf", que tiene una superficie cóncava con una cavidad en la parte delantera de la punta. El área activa para esta sonda se encuentra dentro de la cavidad cóncava y no entrará en contacto con el tejido a menos que el tejido se introduzca en el hueco con succión o la sonda se presione con fuerza contra el área del tejido. Por lo tanto, el área activa útil para este diseño de la cánula es realmente bastante pequeña. Con la tercera generación se diseñaron sondas con anillos en el exterior alrededor del diámetro externo, éstas actúan como un cuchillo ultrasónico al vibrar. La densidad de energía a lo largo del anillo externo es muy alta, lo que resulta en la acción de corte. Esta área activa para esta sonda es en realidad solo una pequeña porción en el centro de la cúpula hemisférica ${ }^{14}$.

En las sondas anilladas y acanaladas de la tecnología de tercera generación, las ranuras actúan para aumentar el área activa para cada sonda y el disco plano pequeño en el centro del extremo hemisférico aumenta el área activa útil de la porción de punta. Este diseño de sonda ha disminuido significativamente la densidad de energía debido a la distribución de la energía total entregada por la sonda a través de un área mucho mayor. La eficiencia de este estilo de sonda es mucho mayor que los diseños de primera o segunda generación ${ }^{14}$.

El efecto de los surcos y anillos se puede examinar para evaluar su uso, de acuerdo a la cantidad de anillos y surcos que tiene, más superficies de contacto tendrá. Por lo tanto, una sonda con más surcos no se deslizará tan bien en el tejido fibroso y es más adecuada para tejidos más blandos. Una sonda con menos surcos tendrá más energía en la parte frontal de la punta y, por lo tanto, penetrará mejor en los tejidos fibrosos ${ }^{14}$.

Por ello surge que el uso correcto del tipo de cánula, o sonda esta determinado por el tipo de tejido, donde se debe utilizar la sonda sin anillos, para estructuras con extremada fibrosis, ejemplo: pacientes con intervenciones previas y retracciones importante. La sonda con un anillo se indica en tejidos con fibrosis. Con dos anillos en moderada fibrosis, y tres anillos en tejidos blandos ${ }^{14}$. La extensión y alcance de la energía de la superficie de la punta de la sonda o zona efectiva alrededor de una punta vibratoria está limitada a aproximadamente 0.5 $\mathrm{mm}$ desde la superficie de la punta. Si el tejido está fuera de esta distancia, generalmente no se verá afectado de ninguna manera. Por lo tanto, el uso efectivo de una sonda ultrasónica vibrante requiere que la punta de la sonda se coloque en contacto con todos los tejidos objetivo $^{14}$.

\subsection{EFECTOS DE LA UAL SOBRE LOS TEJIDOS}

La ventaja de la energía ultrasónica es la selectividad para el tejido. La base para la selectividad tisular es la "fuerza" de los diversos tejidos en relación con la frecuencia de la energía ultrasónica ${ }^{14}$. El nivel de energía ultrasónica se puede ajustar para que los tejidos con las resistencias más bajas estén fragmentadas o emulsionadas (tejidos grasos), mientras que los tejidos con mayores resistencias estén relativamente intactos. Esta es la clave del éxito con la instrumentación ultrasónica ${ }^{30}$.

En la Lipoplastia Asistida por Succión (SAL) el traumatismo es avulsivo asistido por succión, no es selectivo (cualquier cosa arrastrada hacia el puerto de succión se desgarra y se retira),en cambio la Lipoplastia asistida por ultrasonido es selectiva. Cuando se hace correctamente, la lipoplastia asistida por ultrasonido fragmenta el tejido adiposo y crea una emulsión blanda. Las estructuras de colágeno, los vasos y el tejido nervioso se salvan (Figura 6). Debido a que los tejidos o fluidos emulsionados se pueden extirpar más fácilmente con menos traumatismo que con la lipoplastia asistida por succión tradicional, se puede evitar lesionar una mayor parte de la matriz tisular. Por lo tanto, el cuerpo experimenta menos traumatismo lo que da como resultado una curación más rápida, resultados más suaves y menos dolor ${ }^{14}$. Además, la reducción del trauma de la matriz tisular da como resultado una pérdida de sangre significativamente menor, como se ha demostrado al comparar el uso de la tecnología de tercera generación con la aspiración asistida por aspiración labial en la espalda Este estudio encontró 6-7 veces menos sangre en el aspirado para la tecnología de tercera generación vs. $S \mathrm{~L}^{34}$.

Debido a que la instrumentación ultrasónica es menos traumática para la matriz tisular, puede usarse para mejorar la retracción de la piel en la lipoplastia. La retracción cutánea se maximiza cuando la capa grasa superficial se "adelgaza" (hasta $1 \mathrm{~cm}$ por debajo de la parte inferior de la dermis) pero mínimamente traumática, lo que significa que el tejido conjuntivo y la estructura vascular de esta capa superficial permanecen lo menos dañados posible ${ }^{33}$.

Si esta capa superficial se adelgaza con dispositivos de lipoplastia asistida por succión, el resultado es que las estructuras en esta capa se rasgan o se quitan, dejando la piel menos unida a las capas inferiores. Por lo tanto, la piel no experimenta la carga contractiva del tejido conectivo y tiende a asentarse en las capas inferiores y cicatriza en su lugar con muy poca contracción. Alternativamente, si la capa superficial puede ser "desgrasada" con éxito resultando en una reducción de volumen en la capa superficial pero dejando intacta la mayoría de la matriz tisular, entonces la piel se asentará, sanará sujeta a la carga elástica generada durante la curación ${ }^{33}$. El objetivo de la aplicación adecuada de energía ultrasónica en el procedimiento de lipoplastia es reducir el trauma de la matriz tisular, lo que promueve resultados más suaves con más retracción de la piel, sanidad más 
rápida, menos hemorragia y menos dolor. Estos resultados pueden producirse solo con la aplicación adecuada y apropiada de energía ultrasónica. Los dispositivos UAL de generación temprana tenían muchas características de diseño que impedían la logro de estos objetivos como se describe anteriormente en física básica ${ }^{33}$.

\section{MATERIALES Y MÉTODOS}

Se realizó una pesquisa en los meses abril a junio de 2018 sobre informaciones de Lipoplastia asistida por ultrasonido, teniendo como principio de búsqueda, historia de la lipoplastia o liposucción, características de la lipoplastia con ultrasonido, mecanismo de acción del ultrasonido sobre los tejidos. Se enfoca la exploración en tesis, artículos de investigación, revistas científicas, monografías de donde se obtienen los resultados acordes a las necesidades para completar el proyecto.

\section{RESULTADOS}

Se ha demostrado que el ultrasonido en la lipoplastia es utilizado desde hace bastante tiempo debido a sus amplias cualidades de especificidad sobre los tejidos. La evolución y mejor conocimiento de las interacciones con los tejidos por los años de experiencia con el uso de los aparatos determinó que los aparatos de ultrasonidos que generan mayor frecuencia y menor amplitud son más específicos para el tejido adiposo dentro de un rango de oscilación de $22 \mathrm{kHz}$ a $36 \mathrm{kHz}$. Estas modificaciones en los dispositivos hicieron que los efectos de estos sean más eficaces que los empleados anteriormente.

Las tres fundamentos del efecto de los ultrasonidos sobre los tejidos: cavitación, térmico y mecánico, hace que estas propiedades físicos mecánicas propias del ultrasonido, agreguen la principal diferencia a la Lipoplastia Asistida Convencional, aumentado por ello los beneficios del tratamiento y disminuyendo las complicaciones o efectos no deseados habituales de la técnica convencional.

Un importante detalle no menor son los diseños de las cánulas o sondas. Cuando mayor es el diámetro de las cánulas o sondas, mayor sera la lesion de los tejidos sometidos, sin embargo cuanto mas números de anillos o ranuras posea, sera menor el efecto. Por lo que se puede afirmar por tal razón: "La decisión de la cánula o la sonda, esta dada según el grado de acción que se desee, siendo mayor el efecto directamente proporcional al diámetro, e inversamente proporcional al numero de anillos o ranuras".

El objetivo de la aplicación adecuada de energía ultrasónica en el procedimiento de lipoplastia es reducir el trauma de la matriz tisular, lograr resultados más suaves con más retracción de la piel, recuperación más rápida, menos hemorragia y menos dolor. Todos estos beneficios, para aumentar la efectividad del procedimiento a favor del paciente.

\section{DISCUSIÓN}

Como método, la Lipoplastia Asistida por Ultrasonido en el tratamiento de las lipodistrofias, el ultrasonido presenta ventajas superiores sobre la técnica convencional. Aun no es el gold standard en el tratamiento, sin embargo, debido a todo lo mencionado anteriormente en cuanto a las características superiores a la técnica convencional, uno se replantea del porqué aun se sigue utilizando la técnica convencional, será que el costo para adquirir los aparatos, los cuales son bastante altos, sea una barrera para el cirujano plástico "novato" que no puede costearse el valor de la tecnología.

Indefectiblemente el desgaste físico del Cirujano Plastico está totalmente disminuido con el ultrasonido, recordando que el paso de la sonda o cánula se realiza en menor cantidad y con menos fuerza, al contrario de las cánulas clásicas robustas que necesitan alta energía física para lograr su efecto de avulsion y succión. Entonces, porquéno existe una migración de la técnica clásica a la Ultrasónica, o será que a los "seniors" se les dificultad cambiar su metodología de tratamiento, donde quizás estén cómodos y satisfechos con los resultados, para pasar a una nueva técnica que implica una curva de aprendizaje para llegar a los mejores resultados.

Se sabe que con las nuevas generaciones los dispositivos optaron por la utilización de la sonda en vez de las cánulas como se venia haciendo en la 1 ra y 2 da generación de ultrasonidos. Si los resultados con los aparatos de 2 da generación en manos de personas capacitadas a interpretar la cantidad de energía que se debe utilizar de acuerdo al tejido a tratar, eran muy buenos, y ademas con las cánulas solo se necesita un tiempo quirúrgico porque en el mismo acto emulsiona y succiona. Es necesario pasar a la 3ra generación de ultrasonidos los cuales utilizan sondas que requieren mayor tiempo operatorio porque estas solo emulsionan, requiriendo en paso a continuar de cánulas de succión.

\section{CONCLUSIÓN}

Se puede constatar con base en las búsquedas bibliográficas realizadas de la historia de la Lipoplastia o liposucción , características de la lipoplastia asistida con ultrasonido, mecanismo de acción del ultrasonido sobre los tejidos, que los resultados son superiores en cuanto a reducir el trauma de la matriz tisular, lograr resultados más suaves con más retracción de la piel, recuperación más rápida, menos hemorragia, menos dolor, sin producir daño en tejidos adyacentes, y con mínima repercusión en el estado general del paciente.

Con base en los datos obtenidos, la lipoplastia asistida con ultrasonido, podría tratarse de una evolución en el tratamiento de las lipodistrofias, pudiendo sustituir los tratamientos actuales una vez que se opte su utilización como tratamiento primario. 
Se demuestran las ventajas que presenta la lipoplastiaasistida con ultrasonido. Como son la especificidad de su acción sobre el tejido adiposo, actuando exclusiva- mente, sin afectar los tejidos de mayor densidad como son los tejidos, vascular, nervioso, conectivo, muscular y óseo.

\section{BIBLIOGRAFÍA}

1. Flynn TC, Coleman WP III, Field LM, Klein JA, Hanke CW. History of Liposuction. Dermatol Surg. June 2000;26(6):515-20.

2. Asken S. Liposuction Surgery and Autologous Fat Transplantation. East Norwalk, Appleton \& Lange, 1988.

3. Flyn TC. The History of Liposuction. Liposuction Principles and Practices. 2006. 27.

4. Fischer $A$, Fischer $G$. Revised technique for cellulitis fat reduction in riding breeches deformity. Bull Int Acad Cosmet Surg 1977; 2: 40-1.

5. Illouz, Y. Body contouring by lipolysis: a 5-year experience with over 3000 cases. PlastReconstr Surg 1983; 72: 511-24.

6. Coleman WP III. The history of liposuction and fat transplantation in America. Dermatol Clin. 1999;7:723-727.

7. Sattler G, Sommer B, Hanke CW. The history of liposuction. Textbook of the liposuction. 2007.

8. FournierP. BodySculpturing Through Syringe Liposuction and AutologousFat Reinjection. Corona Dee Mar, CA: Samuel RolfInternational, 1987.

9. Klein, J.A. The tumescent technique for liposuction surgery. Am J Cosmet Surg 1987; 4: 236-67.

10. Hanke CW, Bernstein G, Bullock BS. Safety of tumescent liposuction in 15336 patients national survey results. Dermatol Surg 1996; 22:459-62.

11. Forrest JO. Ultrasonic scaling. A fiveyear assessment. $\mathrm{Br}$ Dent J. 1967;122(1):9-14.

12. Kelman C. Phacoemulsification and aspiration: a report of 500 consecutive cases. Am J Ophthalmol. 1973;75(5): 764-8.

13. Cimino WW. The physics of soft tissue fragmentation using ultrasonic frequency vibration of metal probes. Clin Plast Surg. 1999;26(3):447-61.

14. CiminoWW, Shiffman MA. Ultrasound-Assisted Lipoplasty: Basic Physics, Tissue Interactions, and Related Results/Complications. BodyContourning. 2010: 389-398.

15. Scuderi N, Devita R, D'Andrea F, Vonella M. Nuove prospettive nella liposuzione la lipoemulsificazone. Giorn Chir Plast Ricostr ed Estetica. 1987:2(1):33-9.

16. Zocchi ML. New prospectives in liposcultpuring: the ultrasonic energy. 10th ISAPS Congress. Zurich, Switzerland; 1989.

17. Cimino WW. History of Ultrasound-Assisted Lipoplasty. Body Contournig. 2010. 399-402.

18. Topaz, M. Possible longterm complications in ultrasoundassisted lipoplasty induced by sonoluminescence, sonochemistry and thermal effect. Aesthet Surg J 1998; 18: 19-24.
19. Zocchi, M.L. Ultrasonic liposculpturing. Aesthet Plast Surg. 1992;16: 287-98.

20. Zocchi ML. Uttrasonic assisted lipoplasty. Technical refinements and clinical evaluations. Clin Plast Surg. 1996;23:575-598.

21. Rohrich RJ, Morales DE, Krueger JE, et al. Comparative lipoplasty analysis of in vivo-treated adipose tissue. PlastReconstr Surg. 2000;105:2152-2158; discussion 2159.

22. Maxwell GP, Gingrass MK. Ultrasound-assistedlipoplasty: a clinicalstudyof 250 consecutivepatients. Plast ReconstrSurg. 1998;101: 189-202; discussion 203.

23. Rohrich RJ, Beran SJ, Kenkel JM, Adams WPJ, Dispaltro F. Extending the role of liposuction in body contouring with ultrasound-assisted liposuction. PlastReconstr Surg. 1998;101:1090-1102; discussion 1117.

24. Beckenstein MS, Grotting JC. Ultrasound-assisted lipectomy using the solid probe: a retrospective review of 100 consecutive cases. PlastReconstr Surg. 2000;105:2161-2174; discussion 2175.

25. Fodor $P B$, Watson J. Personal experience with ultrasound assisted lipoplasty: a pilot study comparing ultrasound-assisted lipoplasty with traditional lipoplasty. PlastReconstr Surg. 1998;101(4):1103-16.

26. Zocchi ML. Uttrasonic assisted lipoplasty. Clin Plast Surg 1996;23(4):575-98.

27. Troilius C. Ultrasound-assisted lipoplasty: is it really safe? Aesthetic Plast Surg. 1999;23(5):307-11.

28. Tebbetts JB. Minimizing complications of ultrasound assisted lipoplasty: an initial experience with no related complications. PlastReconstr Surg. 1998;102(5): 1690-7.

29. Tebbetts JB. Rapid probe movement ultrasound assisted lipoplasty. Aesthetic Surg J. 1999;19(1):17-23.

30. Cimino WW. Ultrasonic surgery: power quantification and efficiency optimization. Aesthetic Surg J. 2001;21(3): 233-40.

31. Jewell ML, Fodor PB, De Souza Pinto EB, Al Shammari MA. Clinical application of VASER-assisted lipoplasty: a pilot clinical study. Aesthetic Surg J. 2002;22(2):131-46.

32. Cimino WW, Bond LJ. Physics of ultrasonic surgery using tissue fragmentation: part I. Ultrasound Med Biol. 1996; 22(1):89-100.

33. Bond LJ, Cimino WW. Physics of ultrasonic surgery using tissue fragmentation: part II. Ultrasound Med Biol. 1996; 22(1):101-12.

34. Garcia O, Nathan M. Comparative analysis of blood loss in suction-assisted lipoplasty and 3rd generation internal ultrasound assisted lipoplasty. Aesthetic Surg J. 2008; 28:430-5. 